

\section{Interpretation of Renal Quality of Life Profile scores in routine clinical practice: An aid to treatment decision-making}

Dr Nadine Aawar, MPharm, PhD

Research Fellow

South East Wales Trials Unit (SEWTU)

Centre for Trials Research

College of Biomedical \& Life Sciences

Cardiff University, 7th floor Neuadd Meirionnydd

Heath Park, Cardiff

CF14 4YS

Tel: +44 (029) 20687146

Fax: +44 (029) 20687611

E-mail: Aawarn@cf.ac.uk

School of Pharmacy and Pharmaceutical Sciences

Cardiff University, Redwood Building

King Edward VII Avenue

Cardiff

CF10 3NB

Richard Moore

Consultant Physician and Nephrologist

Tairawhiti District Hospital

Gisborne

New Zealand

Stephen Riley

Consultant Nephrologist

Institute of Nephrology

University Hospital of Wales, Cardiff

Sam Salek

Professor of Pharmacoepidemiology

University of Hertforshire, Hatfield, Uk

Director - Institute for Medicines Development, Cardiff

Address for correspondence:

Dr Nadine Aawar,

South East Wales Trials Unit (SEWTU)

Centre for Trials Research

College of Biomedical \& Life Sciences

Cardiff University, 7th floor Neuadd Meirionnydd

Heath Park, Cardiff

CF14 4YS 
Tel: +44 (029) 20687146

Fax: +44 (029) 20687611

E-mail: Aawarn@cf.ac.uk 


\section{INTRODUCTION}

The ultimate goal of medical care is to improve patients' functional behaviour, both physical and psychological. In recent years, the value of quality of life information in clinical trials has been widely demonstrated. However, the abundance of health-related quality of life (HRQoL) instruments available, each with different units of measurement, renders interpretation even more difficult and challenging for clinicians and healthcare providers (1). One suggestion for improving the meaningfulness of HRQoL measures in clinical practice is the use of the anchor-based technique (2). The Renal Quality of Life Profile (RQLP) is a user-friendly self-administered modality independent instrument. Its reliability and validity have been demonstrated (3). Basic information about interpretation of the scores is not sufficient and when used to signal a change in patient functioning in response to a new treatment, the magnitude of the change is often difficult for clinicians and healthcare planners to interpret (4). Several different approaches to interpret HRQoL score have been used (2). In this study, the anchor-based approach was employed, since it is most appropriate for short, relatively simple HRQoL instruments. This approach is based on a patient global rating question (GQ) which measures patients' views of their overall impairment of HRQoL due to chronic kidney failure (CKF). The GQ can then be used alongside a HRQoL instrument. The use of the anchor-based technique to determine categorization for HRQoL measures has been described previously in conjunction with the Dermatology Life Quality Index (DLQI) (5), in the Rhinoconjunctivitis Quality of Life Questionnaire (RQLQ) (6) and Skindex-29 Questionnaire (7). This methodology will facilitate instant interpretation of quality of life scores during consultation and will provide support for clinicians to remove apprehension about the use and importance of HRQoL in treatment decision-making. The aim of this study was therefore, to assign clinical 
significance to RQLP scores by establishing ranges of scores or categories within the scale of the measure.

\section{SUBJECTS AND METHODS}

\section{Study design}

This was a prospective study for which a consecutive sample of 260 patients was recruited. Data was collected from home and unit haemodialysis, pre-dialysis, peritoneal dialysis and transplant outpatient clinics, at the University Hospital of Wales, United Kingdom.

\section{Patients}

All patients aged 18 years and above diagnosed with chronic renal failure were recruited into the study. Patients whose physical condition prevented them from completing the questionnaire were excluded. Eligibility of patients for the study was assessed jointly by the outpatient clinics coordinator and the respective physician (s).

\section{Study instruments}

The Renal Quality of Life Profile (RQLP) was used to collect HRQoL data from patients with chronic renal failure. It is an original questionnaire based on constructs representing renal patient's own quality of life determinants. Two thousand six hundred statements related to the quality of life areas most affected by renal conditions were generated from patients receiving different treatment modality, nephrologists, renal transplant surgeons and renal nurses. The RQLP is a condition specific and modality independent quality of life instrument which measures individual perceptions of the impact of disease and treatment on patients' quality of life with different treatment modality (i.e. pre-dialysis, haemodialysis, peritoneal dialysis and transplant). The instrument consists of 43 items, with a 5-point Likert response scale describing health-related dysfunctional behaviours that are specific to chronic renal failure patients and is grouped into five categories [eating and drinking(3 
items), physical ability (16 items), leisure time (3 items), daily life (13 items) and impact of treatment ( 8 items)]. The sum of the scores provides a value between 0 (no impact on the HRQoL of the patient) and 172 (maximum impact on the HRQoL of the patient). The questions are easily understood, with an average completion time of 5-7 minutes (3). The RQLP measurement properties have been established and number of studies been carried out as part of its developmental work to demonstrate its reliability and sensitivity. The reliability of the subscales of the RQLP was tested using Cronbach's alpha, and all showed good internal consistency; eating/drinking $(\alpha=0.88)$; physical activities $(\alpha=0.90)$; leisure time $(\alpha=0.85)$; psychosocial activities $(\alpha=0.84)$ and impact of treatment $(\alpha=0.75)$ (3). The construct validity of the RQLP was explored by comparing the RQLP with the United Kingdom Sickness Impact Profile (UKSIP). High correlations were achieved, in particular, among the common categories (rs=0.70). The RQLP was compared with SF-36 similarly high levels of correlations were achieved in reflecting patients' assessment of their quality of life ( $r s=0.58$ to 0.75 ). The RQLP is also sensitive to deterioration as well as improvement (8).Therefore, robustness of the RQLP, a modality independent instrument, renders it useful in routine clinical practice.

\section{Anchor-based approach}

There are two main approaches for interpreting HRQoL, the anchor-based and the population-based technique. The anchor-based approach (2) used for this study has not previously been applied to CKD specific HRQoL instruments; however a similar method has been shown to be successful using a dermatology HRQoL measure (5). Our study met the two cardinal requirements of this approach (9). First, the GQ was interpretable and understandable by the study subjects. Second, a strong correlation was demonstrated 
between the target (RQLP) and the anchor (GQ). A global question (GQ) was used in order to examine the relationship between patients' evaluation of their overall HRQoL and the multi-dimensional RQLP scores. Following previous studies which have used anchor based approach (5) the GQ asks: "Over the past week, how much has your kidney problem affected your life?" The five possible response options are: extremely large effect on my life (4 points); very large effect on my life (3 points); moderate effect on my life (2 points); a small effect on my life (1 point); and no effect on my life (0 points).

Implementation of quality of life assessment in routine practice and the use of score banding system

Quality of life assessment was integrated into the routine process of a renal outpatient clinic at the Royal Gwent Hospital, Newport, Wales, United Kingdom. This involved a prospective evaluation of all medical consultations taking place during a fifty-day period incorporating the patients' self-report of quality of life into their routine clinical assessment process. At the clinic, patients were asked to complete and score the RQLP in the waiting room before seeing the clinicians. The pre-consultation QoL information was passed onto the examining clinicians (five in total) and was used during their consultation with the patient. The categorization of the RQLP scores was provided at the bottom of the first page for ease of interpretation of scores by the clinicians. The clinicians recorded their own response with respect to the aspects of the patients' reported quality of life that they found helpful and their influence on change of treatment or new interventions, if any, arising from that process. They were also asked to complete three questions in a tick box format concerning the value and use of the quality of life information during consultation.

\section{Statistical analysis}

Data were entered and processed using SPSS version 16 for windows. The Mann-Whitney $\mathrm{U}$ test was used for gender comparison. The Spearman rank correlation coefficient was 
used to examine the correlation between the RQLP scores and the GQ scores. The proposed "categories" were devised using the mean, median and mode of the GQ scores mapped against each RQLP score and the ICC coefficient of agreement was calculated for each set.

\section{RESULTS}

\section{Demographic characteristics of the study participants}

A total of 260 patients with chronic renal failure (haemodialysis $n=69$, Continuous ambulatory peritoneal dialysis $(\mathrm{CAPD}) \mathrm{n}=65$, pre-dialysis $\mathrm{n}=57$ and transplant $\mathrm{n}=69$ ) were recruited from the renal outpatient clinics. The patients' mean age was 58.4 years with a median of 59 years and a range of 21 to 90 years. There were $165(63.5 \%)$ male and $95(36.5 \%)$ female patients. The mean age of males (58.5) was similar to that of the females (58.3) despite the wider age range for females (21-90 years) compared to males (22-86).

\section{The RQLP scores}

The RQLP scores covered a good range between the minimum score of zero and a maximum possible score of 172 with higher score representing more impairment. The overall mean score was $67.2(\mathrm{SD}=41.5)$ with a median of $61($ range $=0-169)$. The mean score for males was 60.9 ( $\mathrm{SD}=38.6$ ) with a median of 57 (range=0-158). For females, HRQoL was more impaired with a mean of $78.4(\mathrm{SD}=44)$ and a median of 73 (range=0-169) reflecting a wider range of an 11 score difference. The Mann-Whitney U Test showed a significant difference $(\mathrm{p}<0.01)$ between men (median=57, $\mathrm{n}=165)$ and women $($ median=73, $\mathrm{n}=95)$ for the RQLP.

\section{The GQ scores}


The patients selected all five possible GQ scores with the middle option i.e. score of 2 ("moderate effect") being the most frequently scored, as expected for this type of scale (10). The GQ mean scores for males and females were 1.64 and 1.92, respectively. The median for both groups was 2 ("moderate effect"). The Mann-Whitney U Test showed no significant difference for the GQ scores $(\mathrm{p}=0.14)$. The spearman rank correlation coefficient showed a strong correlation between the RQLP scores and the GQ scores ( $r=$ $0.73, \mathrm{p}<0.01)$.

\section{The development of a categorization system for the RQLP scores}

The RQLP scores and their corresponding GQ scores for all patients were categorised with five point intervals, as well as the mean, median and mode of the GQ scores for that specific RQLP interval. Subsequently, the RQLP scores were grouped into five categories, each corresponding to a level of impairment as indicated by the GQ (Figure 1). There was a reasonable amount of overlap between some of the possible discrete categories where a number of RQLP scores could have fitted into one of two categories. A number of "grey" areas with multiple potential overlap led to a number of different sets of score categorizations being produced. The Intraclass Correlation Coefficient (ICC) was then calculated to determine the level of agreement between these different groups. Table 1 shows the different category selections that were proposed with corresponding ICC values that were calculated for each set of categories. All the proposed categories had an ICC value of 0.8 or greater out of the maximum possible value of 1 , which is within the acceptable range and indicate strong agreement (11). The proposed RQLP categories of 020, 21-51, 52-93, 94-134, and 135-172 (Set A), showed well balanced data (i.e. the majority of the patients fitted within the category that would have been predicted from their GQ scores and a very small percentage of patients did not fit within the category that would 
have been predicted from their GQ scores. This is largely the nature of anchor based approach) (Table 2), whilst sets B, C, D, E, F and G showed problems in the distribution of patients along the GQ and the corresponding RQLP scores. The categorization system that was therefore proposed for adoption is 0-20 (no effect on patient' life), 21-51 (small effect on patient's life), 52-93 (moderate effect on patient's life), 94-134 (very large effect on patient's life) and 135-172 (extremely large effect on patient's life) (Set A).

\section{Clinicians' attitude towards the value and use of the RQLP categorization system during consultations}

The acceptability and practicality of the RQLP banding scores presented in the previous section above was tested in a secondary care renal outpatient clinic. A total of 5 renal consultants were recruited into the study who used the RQLP banding system in their consultation with 46 patients. The 46 consultations had a response from the doctors regarding the use of the RQLP categorization system. Seventy-four percent of the clinicians found the categorization of the RQLP helpful in interpreting the scores. Twenty-two percent of the consultations reported to have used the RQLP information provided during the consultation. Seven percent reported that the RQLP information influenced their treatment decision- making.

\section{DISCUSSION}

Health-related quality of life measurement has become increasingly important in clinical research, especially for such chronic conditions as kidney disease (12). However, whilst it is generally agreed that it is an important area of research, it still remains a difficult concept to translate into use in routine clinical practice. Reliability and validity of many of the measures used to assess HRQoL in chronic kidney failure has been established using 
classical test theory. Due to the complexity of many of these measures in both calculation of a score and in particular interpreting that score, HRQoL measures have not yet been accepted in clinical practice. Therefore, the purpose of this study was to make HRQoL scores more meaningful by establishing ranges of scores or categorization within the scale of the measure. This approach could provide clinicians with both a rapid overall view of the patients' HRQoL in addition to the detection of small but meaningful changes in clinical status over time.

It was also important that the RQLP and the GQ were closely correlated which was shown in this study. Although there was an alternative set of categories that had a higher ICC value compared to the proposed category, there are other factors to take into consideration. For example, the majority of the patients fitted within the category that would have been predicted from their GQ score which made the data set matrix well balanced. A very small percentage of patients did not fit within the category that would have been predicted from their GQ score. A larger patient population would have given a better range of scores, thus eliminating this problem.

Using a patient's quality of life information in clinical practice can offer a number of benefits leading to optimised treatment decision-making. For example, it can be used to prioritise problems, facilitate communication, screen for potential risks, identify preferences, monitor changes or response to treatment and train new staff. They can also be used in clinical audit and in clinical governance. The first five of these are of immediate value in the clinical encounter, while the last three contribute to training, reviewing and improving care in the future which in turn contributes to the quality of decision-making (13). Quality of life measures are not a substitute for measuring clinical outcomes associated with disease but are an adjunct to them. Being able to interpret the RQLP scores 
may be helpful to clinicians to detect unrecognized problems, monitor the impact of treatment and appreciate patient priorities (14). Overall the provision of HRQoL information in clinical practice may facilitate communication, lead to improvements in patient's health status and satisfaction with their care (15). Consequently, this will translate into a "patient-centred" clinical practice.

Using a categorization system will allow harmonisation of treatment strategies between clinicians, for example, results from this study show that scores over a value of 93 describe patients who are experiencing a poor HRQoL, in other words "those at risk". For patients with a score greater than this value, there would be strong evidence that active intervention is required and clinicians will be able to target these patients with appropriate treatment as necessary.

Finally, this approach is not intended to dilute the significance of independent HRQoL scores, but instead to provide a complementary process. This will allow healthcare professionals to obtain a relatively rapid overall observation of a patient's HRQoL from the categorization in addition to a more thorough examination through the detailed HRQoL measure when required. For example, when a patient presents with a score of over 93, closer inspection of the HRQoL profile will allow the clinician to identify specific aspects of the patient's life that are most severely affected by the disease, thus allowing a more targeted treatment strategy. However, healthcare professionals will be comfortable with such scores only when they become familiar with them (16) which will require their routine use in clinical research and practice (17). It would however be of interest to clinicians to compare the anchor based approach used in this study with linking HRQoL scales such as RQLP directly with clinical relevant markers. 
A larger sample size would have given a more uniform distribution of the RQLP scores. It is therefore possible that certain levels of disease severity were not represented in the range of scores. The fact that this is a single centre study could be considered as a limitation, however, the RQLP is a modality independent instrument and the centre where the patients were recruited provides renal services to over two million people living within South Wales.

\section{DISCLOSURE}

All the authors declared no competing interests 


\section{REFERENCES}

1. Crosby RD, Kolotkin RL, Williams GR. Defining clinically meaningful change in health related quality of life. Value Health 2001; 4: 177.

2. Lydick E, Epstein RS. Interpretation of quality of life changes. Qual Life Res 1993; 2: 221-226.

3. Salek MS. Renal Quality of Life Profile User Manual Oxford Outcomes, Oxford; 2000.

4. Testa MA. Interpretation of quality-of-life outcomes: issues that affect magnitude and meaning. Med Care 2000; 38: 166-174.

5. Hongbo Y, Thomas CL, Harrison MA, Salek MS, Finlay AY. Translating the Science of Quality of Life into Practice: What Do Dermatology Life Quality Index Scores Mean?. J Invest Dermatol 2005; 125: 659-664.

6. Juniper EF, Guyatt GH, Griffith LE, Ferrie PJ. Interpretation of rhinoconjunctivitis quality of life questionnaire data. J Allergy Clinic Immun 1996; 98: 843-845.

7. Prinsen CAC, Lindeboom R, Sprangers MAG, Legierse CM, de Korte J. Health-Related Quality of Life Assessment in Dermatology: Interpretation of Skindex-29 Scores Using Patient-Based Anchors. J Invest Dermatol 2010; 130: 1318-1322.

8 Salek S. Quality of life in patients with end-stage renal disease. J Appl Ther 1999; 2: 163-170. 
9. Guyatt GH, Osoba D, Wu AW, Wyrwich KW, Norman GR. Methods to explain the clinical significance of health status measures. Mayo Clin Proc 2002; 77: 371-383.

10. Edwards, M. L. \& Smith, B. C. (2011). The effects of the neutral response option on the extremeness of participant responses. Incite, 6. Retrieved from http://blogs.longwood.edu/incite/2014/05/07/the-effects-of-the-neutral-response-optiononthe-extremeness-of-participant-responses

11. Rosner B. Fundamentals of biostatistics. $7^{\text {th }}$ ed. New York, NY: Duxbury Press; 2010

12. Rettig RA, Sadler JH, Meyer KB, Wasson JH, Parkerson Jr GR, Kantz B, et al. Assessing health and quality of life outcomes in dialysis: a report on an Institute of Medicine workshop. Am J kidney dis 1997; 30: 140-155.

13. Higginson IJ, Carr AJ. Using quality of life measures in the clinical setting. BMJ 2001; 322: $1297-1300$.

14. Solari A. Role of health-related quality of life measures in the routine care of people with multiple sclerosis. Health Qual life Out 2005; 3 (1): 16.

15 Velikova G, Booth L, Smith AB, Brown PM, Lynch P, Brown JM, et al. Measuring quality of life in routine oncology practice improves communication and patient wellbeing: a randomized controlled trial. J Clin Oncol 2004; 22: 714-724. 
16. Deyo RA, Patrick DL. The significance of treatment effects: the clinical perspective. Med Care 1995; 33: AS286-AS291.

17. Chren MM. Measurement of vital signs for skin diseases. J Invest Dermatol. 2005; 125(4): viii-ix. 


\section{Titles and legends}

Table 1 Different possible sets of categorization for the Renal Quality of Life scores

\begin{tabular}{|c|c|c|c|c|c|c|}
\hline \multicolumn{6}{|c|}{ Allocation of RQLP scores into categories } & \multirow[b]{2}{*}{ ICC } \\
\hline Bands & $\mathbf{0}^{\mathbf{a}}$ & $\mathbf{1}^{\mathbf{b}}$ & $2^{\mathrm{c}}$ & $3^{\mathbf{d}}$ & $4^{e}$ & \\
\hline Set A & $0-20$ & $21-51$ & $52-93$ & $94-134$ & $135-172$ & 0.80 \\
\hline Set B & $0-30$ & $31-55$ & $56-100$ & $101-130$ & $131-172$ & 0.90 \\
\hline Set C & $0-30$ & $31-60$ & $61-100$ & $101-130$ & $131-172$ & 0.92 \\
\hline Set D & $0-30$ & $31-55$ & $56-90$ & $91-130$ & $131-172$ & 0.94 \\
\hline Set $\mathbf{E}$ & $0-27$ & $28-54$ & $55-95$ & $96-122$ & $123-172$ & 0.95 \\
\hline Set $\mathbf{F}$ & $0-29$ & $30-56$ & $57-96$ & $97-125$ & $126-172$ & 0.97 \\
\hline Set G & $0-29$ & $30-56$ & $57-92$ & $93-121$ & 122 to 172 & 0.99 \\
\hline
\end{tabular}

${ }^{a}$ No effect on patients' life, ${ }^{\mathrm{b}}$ Small effect on patients' life, ${ }^{\mathrm{c}}$ Moderate effect on patients' life,

${ }^{d}$ Very large effect on patients' life, ${ }^{\text {e }}$ Extremely large effect on patients' life 
Table 2 Distribution of Global Question scores for the proposed Renal Quality of Life categories (Set A)

\begin{tabular}{l|lllll|r}
\hline $\begin{array}{l}\text { RQLP categories } \\
\text { (Scores) }\end{array}$ & \multicolumn{5}{|c|}{ GQ Score $^{\text {Totals }}$} \\
\cline { 2 - 6 } & $\mathbf{0}^{\mathbf{a}}$ & $\mathbf{1}^{\mathbf{b}}$ & $\mathbf{2}^{\mathbf{c}}$ & $\mathbf{3}^{\mathbf{d}}$ & $\mathbf{4}^{\mathbf{e}}$ & \\
\hline $\begin{array}{l}\text { Category 0 } \\
(\text { Scores 0-20) }\end{array}$ & 30 & 5 & 0 & 0 & 0 & 35 \\
$\begin{array}{l}\text { Category 1 } \\
(\text { Scores 21-51) }\end{array}$ & 16 & 27 & 21 & 1 & 1 & 66 \\
$\begin{array}{l}\text { Category 2 } \\
(\text { Scores 52-93) }\end{array}$ & 7 & 22 & 36 & 18 & 4 & 87 \\
$\begin{array}{l}\text { Category 3 } \\
(\text { Scores 94-134) }\end{array}$ & 2 & 4 & 15 & 24 & 9 & 54 \\
$\begin{array}{l}\text { Category 4 } \\
(\text { Scores 135-172) }\end{array}$ & 0 & 0 & 1 & 4 & 13 & 18 \\
\hline Totals & 55 & 58 & 73 & 47 & 27 & 260 \\
\hline
\end{tabular}

$\mathrm{ICC}=0.80$

${ }^{\mathrm{a}}$ No effect on patients' life, ${ }^{\mathrm{b}}$ Small effect on patients' life, ${ }^{\mathrm{c}}$ Moderate effect on patients' life,

${ }^{\mathrm{d}}$ Very large effect on patients' life, ${ }^{\mathrm{e}}$ Extremely large effect on patients' life 
Figure 1 Relationship between the Renal Quality of Life score and the mean, median and mode of the global question (GQ) score

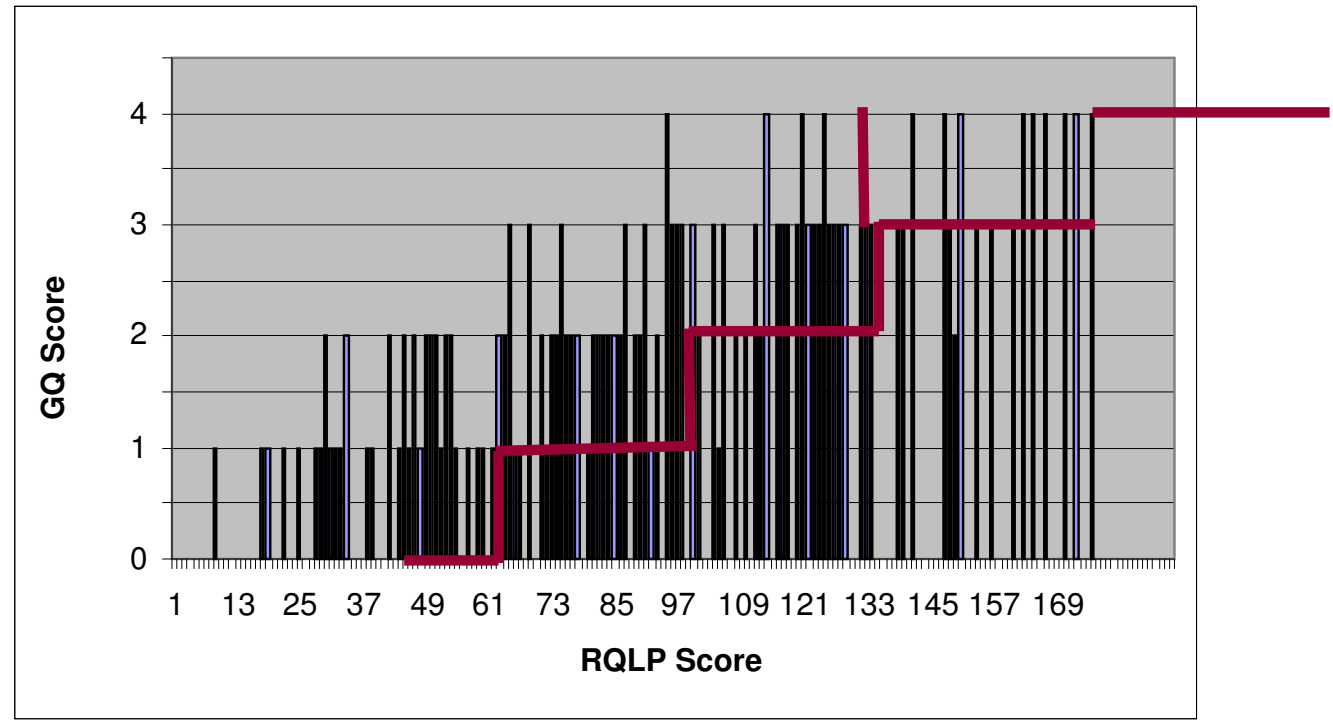

